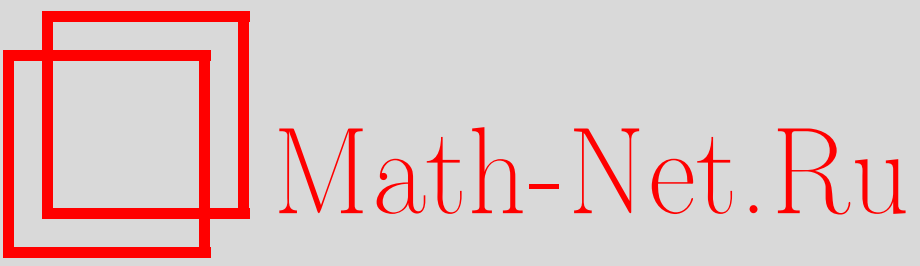

В. П. Радченко, Е. А. Андреева, А. В. Никишаев, Структурная модель ползучести нелинейно-упругого микронеоднородного материала в условиях сложного напряженного состояния, Вестн. Сам. гос. техн. ун-та. Сер. Физ.-мат. науки, 2010, выпуск 1(), 60-70

DOI: https://doi.org/10.14498/vsgtu772

Использование Общероссийского математического портала Math-Net.Ru подразумевает, что вы прочитали и согласны с пользовательским соглашением

http://www.mathnet.ru/rus/agreement

Параметры загрузки:

IP: 52.6 .47 .48

26 апреля 2023 г., 15:14:29 
УДК 539.376

\title{
СТРУКТУРНАЯ МОДЕЛЬ ПОЛЗУЧЕСТИ НЕЛИНЕЙНО- УПРУГОГО МИКРОНЕОДНОРОДНОГО МАТЕРИАЛА В УСЛОВИЯХ СЛОЖНОГО НАПРЯЖЕННОГО СОСТОЯНИЯ
}

\author{
В. П. Радченко, Е.А. Андреева, А.В. Никишаев
}

Самарский государственный технический университет, 443100, Самара, ул. Молодогвардейская, 244.

E-mails: radch@samgtu.ru, andreevaSamara25@yandex.ru, anton.nik@gmail.com

\begin{abstract}
Предложена структурная модель микронеоднородного нелинейно-упругого материала для описания ползучести при сложном напряженном состоянии. Показано, что для такого класса материалов наблюдается эбфект влияния деформачии ползучести на упругую дебормачию, при этом происходит не только изменение модуля вектора нелинейных упругих дебормащий в пространстве дебормаций вследствие ползучести, но и его поворот. Приведень результаты вариативных расчётов, выполнен их анализ.
\end{abstract}

Ключевые слова: нелинейно-упругий материал, ползучесть, структурная модель, сложное напряженное состояние, дрейб упругой дебормаиии.

1. Постановка задачи. Наряду с конструкционными и природными материалами с линейными упругими свойствами, в поле зрения механиков находятся и ряд микронеоднородных биокомпозитных материалов с неклассическими реологическими свойствами, для которых наблюдается эффект влияния деформации ползучести на нелинейно-упругую деформацию, которая испытывает дрейф при выдержках под нагрузкой. Примером такого материала может служить костная ткань. Экспериментальные $[1,2]$ и теоретические [3-7] исследования одноосной ползучести такого рода материалов показали, что мгновенно-упругая деформация этого материала при нагрузке $e^{\mathrm{H}}$ при $t=0+0$ и полной разгрузке после ползучести при действии постоянного напряжения $e^{\mathrm{p}}$ существенно отличаются друг от друга, причем может выполняться как соотношение $e^{\mathrm{p}}>e^{\mathrm{H}}$, так и $e^{\mathrm{p}}<e^{\mathrm{H}}$.

Систематический анализ этого явления для одноосного напряженного состояния на основании структурной модели микронеоднородной среды типа обобщенной двухэлементной нелинейной модели Максвелла с нелинейными упругими и вязкими элементами был выполнен в работах $[6,7]$. Основной результат этих исследований сформулирован в виде двух следующих утверждений.

УтвеРЖДЕниЕ 1. Если локалъные элементы двухэлементной обобщенной модели типа Максвелла удовлетворяют линейному закону упругости, то упругая деформачия на макроуровне не зависит от дебормачии ползучести.

УТВЕРЖДЕнИЕ 2. Если хотя бы один локальный элемент двухэлементной обобщенной модели следует нелинейному закону упругости, то упругая макродеформащия зависит от накопленной деформации ползучести.

Владимир Павлович Радченко (д.ф.-м.н., проф.), зав. кафедрой, каф. прикладной математики и информатики. Елена Анатолъевна Андреева, аспирант, каф. прикладной математики и информатики. Антон Владимирович Никишаев, инженер-программист, каф. прикладной математики и информатики. 
В работе [8] дальнейшее исследование позволило детализировать эффект влияния ползучести на нелинейно-упругую деформацию в одноосном случае для режима нагружения «нагрузка - разгрузка» и установить следующее

1) для нелинейно-упругих материалов происходит медленный дрейф диаграммы упругого деформирования $\langle e\rangle-\langle\sigma\rangle$ вследствие ползучести, при этом весь спектр диаграмм расположен между двумя стационарными состояниями, а именно диаграммой при $t=0$ и диаграммой, соответствующей асимптотическому состоянию структурной модели под нагрузкой при $t \rightarrow \infty$ (стадии установившейся ползучести);

2) в процессе дрейфа мгновенно-упругая деформация проявляет одновременно свойства механической памяти, поскольку в процессе разгрузки образца (при $t \rightarrow \infty$ ) происходит полное восстановление первоначальных упругих свойств, а также вязкоупругости, поскольку мгновенноупругая деформация неявно (через накопленную деформацию ползучести) зависит от времени.

Выполненный краткий анализ проблемы дрейфа упругой деформации вследствие ползучести для микронеоднородных материалов, моделируемых обобщенной двухэлементной моделью типа Максвелла, показывает, что всесторонне понять и описать эффект влияния ползучести на мгновенно-упругую деформацию можно лишь с позиций механики микронеоднородных сред. Однако все вышеизложенное касалось лишь одноосного напряженно-деформированного состояния (растяжение образца). Естественно возникает вопрос об обобщении, уточнении и анализе полученных результатов для условий сложного напряженного состояния. Поэтому целями настоящей работы являются:

1) разработка структурной модели ползучести нелинейно-упругого материала, описывающей эффект влияния ползучести на упругую деформацию, в условиях сложного напряженного состояния;

2) исследование и анализ ползучести нелинейно-упругих материалов в условиях сложного напряженного состояния.

2. Структурная модель ползучести нелинейно-упругого материала в условиях сложного напряженного состояния. Рассмотренные в [5-8] структурные модели для описания одноосного растяжения на основе обобщенных моделей Максвелла применить к анализу в условиях сложного-напряженного состояния проблематично. Поэтому в настоящей работе используется модификация структурной модели, предложенной в [9], согласно которой микронеоднородный материал моделируется системой хаотически ориентированных однородных стержней (локальных элементов) одинаковой длины, работающих на растяжение - сжатие. Каждый локальный элемент этой системы наделяется свойствами нелинейной упругости (в отличии от модели [9], где использован закон линейной упругости) и нелинейной вязкости.

Рассмотрим материал, на который действует тензор напряжений, приведенный к главным осям $O X Y Z$. Ориентация элементарных стержней (локальных элементов) задается двумя сферическими углами $\theta$ и $\varphi(0 \leqslant \theta \leqslant \pi / 2$, $0 \leqslant \varphi \leqslant 2 \pi)$, причем $\theta$ определяет угол между направлением элементарного стержня и осью $O X$. Через $\sigma(\theta, \varphi)$ обозначим напряжение (микронапряжение), возникающее в локальном элементе; $\varepsilon(\theta, \varphi)$ - деформацию (микродеформацию) локального элемента, $\left\langle\sigma_{x}\right\rangle,\left\langle\sigma_{y}\right\rangle,\left\langle\sigma_{z}\right\rangle$ - макронапряжения (глав- 
ные напряжения); $\left\langle\varepsilon_{x}\right\rangle,\left\langle\varepsilon_{y}\right\rangle,\left\langle\varepsilon_{z}\right\rangle$ - макродеформации (главные деформации).

Тогда уравнения состояния локального элемента примут вид:

$$
\begin{gathered}
\varepsilon(\theta, \varphi, t)=e(\theta, \varphi, t)+p(\theta, \varphi, t), \\
e(\theta, \varphi, t)=\frac{\sigma(\theta, \varphi, t)|\sigma(\theta, \varphi, t)|^{n-1}}{E_{\mathrm{M}}}, \\
\dot{p}(\theta, \varphi, t)=a \sigma(\theta, \varphi, t)|\sigma(\theta, \varphi, t)|^{m-1},
\end{gathered}
$$

где $E_{\mathrm{M}}, n$ - параметры аппроксимации упругой деформации; $a, m-$ параметры аппроксимации деформации вязкого течения. Уравнения равновесия

$$
\begin{aligned}
& \left\langle\sigma_{x}\right\rangle=\frac{1}{\pi} \int_{0}^{\pi / 2} \cos ^{2} \theta \sin \theta d \theta \int_{0}^{2 \pi} \sigma(\theta, \varphi) d \varphi, \\
& \left\langle\sigma_{y}\right\rangle=\frac{1}{\pi} \int_{0}^{\pi / 2} \sin ^{3} \theta d \theta \int_{0}^{2 \pi} \sigma(\theta, \varphi) \cos ^{2} \varphi d \varphi, \\
& \left\langle\sigma_{z}\right\rangle=\frac{1}{\pi} \int_{0}^{\pi / 2} \sin ^{3} \theta d \theta \int_{0}^{2 \pi} \sigma(\theta, \varphi) \sin ^{2} \varphi d \varphi,
\end{aligned}
$$

гипотеза однородности деформации по объему

$$
\left\langle\varepsilon_{x}\right\rangle=\varepsilon(0,0), \quad\left\langle\varepsilon_{y}\right\rangle=\varepsilon(\pi / 2,0), \quad\left\langle\varepsilon_{z}\right\rangle=\varepsilon(\pi / 2, \pi / 2)
$$

и уравнение совместности деформаций

$$
\varepsilon(\theta, \varphi)=\left\langle\varepsilon_{x}\right\rangle \cos ^{2} \theta+\left\langle\varepsilon_{y}\right\rangle \sin ^{2} \theta \cos ^{2} \varphi+\left\langle\varepsilon_{z}\right\rangle \sin ^{2} \theta \sin ^{2} \varphi
$$

будут такими же, как в [9].

Таким образом, структурная модель ползучести нелинейно-упругого материала задаётся соотношениями (1)-(6).

3. Идентификация параметров структурной реологической модели.

$1^{\circ}$. Упругая область. Для идентификации параметров $n$ и $E_{\mathrm{M}}$, описывающих упругие свойства локальных элементов, рассмотрим одноосное растяжение образца из нелинейно-упругого материала $\left(\left\langle\sigma_{x}\right\rangle \neq 0,\left\langle\sigma_{y}\right\rangle=\left\langle\sigma_{z}\right\rangle=0\right.$, $\left.\left\langle\varepsilon_{y}\right\rangle=\left\langle\varepsilon_{z}\right\rangle\right)$. Предположим, что закон нелинейной упругости на макроуровне имеет вид

$$
\left\langle\varepsilon_{x}\right\rangle=\frac{1}{E}\left\langle\sigma_{x}\right\rangle\left|\left\langle\sigma_{x}\right\rangle\right|^{n-1}
$$

где $E$ и $n$-макропараметры, т. е. полагается, что показатели нелинейности на микро- и макроуровне совпадают.

Запишем в упругой области при одноосном растяжении уравнения равновесия

$$
\begin{gathered}
\left\langle\sigma_{x}\right\rangle=2 \int_{0}^{\frac{\pi}{2}} \sigma(\theta, \varphi) \cos ^{2} \theta \sin \theta d \theta, \\
\int_{0}^{\frac{\pi}{2}} \sigma(\theta, \varphi) \sin ^{3} \theta d \theta=0
\end{gathered}
$$


и совместности деформаций $\left(\left\langle\varepsilon_{y}\right\rangle=\left\langle\varepsilon_{z}\right\rangle\right)$

$$
\varepsilon(\theta, \varphi)=\left\langle\varepsilon_{x}\right\rangle \cos ^{2} \theta+\left\langle\varepsilon_{y}\right\rangle \sin ^{2} \theta .
$$

Тогда, учитывая, что $p(\theta, \varphi, t)=0$, из $(1),(2)$ и $(10)$ имеем

$$
\frac{1}{E_{\mathrm{M}}} \sigma(\theta, \varphi)|\sigma(\theta, \varphi)|^{n-1}=\left\langle\varepsilon_{x}\right\rangle \cos ^{2} \theta+\left\langle\varepsilon_{y}\right\rangle \sin ^{2} \theta
$$

Откуда

$$
\sigma(\theta, \varphi)=\left(E_{\mathrm{M}}\right)^{\frac{1}{n}}\left(\left\langle\varepsilon_{x}\right\rangle\right)^{\frac{1}{n}}\left(\cos ^{2} \theta+\frac{\left\langle\varepsilon_{y}\right\rangle}{\left\langle\varepsilon_{x}\right\rangle} \sin ^{2} \theta\right) \cdot\left|\cos ^{2} \theta+\frac{\left\langle\varepsilon_{y}\right\rangle}{\left\langle\varepsilon_{x}\right\rangle} \sin ^{2} \theta\right|^{\frac{1}{n}-1} .
$$

Вводя обозначение

$$
C=\cos ^{2} \theta+\frac{\left\langle\varepsilon_{y}\right\rangle}{\left\langle\varepsilon_{x}\right\rangle} \sin ^{2} \theta
$$

и подставляя (11) в (8), (9), получаем систему уравнений

$$
\begin{gathered}
\left\langle\sigma_{x}\right\rangle=2 \int_{0}^{\frac{\pi}{2}}\left(E_{\mathrm{M}}\right)^{\frac{1}{n}}\left(\left\langle\varepsilon_{x}\right\rangle\right)^{\frac{1}{m}} C|C|^{\frac{1}{n}-1} \cos ^{2} \theta \sin \theta d \theta, \\
\int_{0}^{\frac{\pi}{2}} C|C|^{\frac{1}{n}-1} \sin ^{3} \theta d \theta=0 .
\end{gathered}
$$

Сначала решается численно второе уравнение системы (13) относительно неизвестной величины $y=\left\langle\varepsilon_{y}\right\rangle /\left\langle\varepsilon_{x}\right\rangle(-1<y<0)$, тем самым определяется величина $C$ в (12). Далее, зная величину $C$ и используя закон макроупругости (7), из первого уравнения (13) устанавливаем связь между макро- и микропараметрами $E$ и $E_{\mathrm{M}}$ :

$$
E_{\mathrm{M}}=\left(\frac{2}{E} \int_{0}^{\frac{\pi}{2}} C|C|^{\frac{1}{n}-1} \cos ^{2} \theta \sin \theta d \theta\right)^{-n} .
$$

$2^{\circ}$. Область ползучести. Для идентификации параметров нелинейной вязкости структурного элемента в (7) необходимо иметь серию одноосных кривых позучести с участком установившейся ползучести, которая описывается законом нелинейной ползучести вида (при $t \rightarrow+\infty$ )

$$
\left\langle\dot{\varepsilon}_{x}\right\rangle=A\left\langle\sigma_{x}\right\rangle\left|\left\langle\sigma_{x}\right\rangle\right|^{m-1}
$$

где $A$ и $m$-макропараметры. Из (15) и (3) следует, что нелинейности для скорости вязкого течения на микро- и макроуровне полагаются равными.

Продифференцируем уравнение совместности деформаций (10) по времени:

$$
\dot{\varepsilon}(\theta)=\left\langle\dot{\varepsilon}_{x}\right\rangle \cos ^{2} \theta+\left\langle\dot{\varepsilon}_{y}\right\rangle \sin ^{2} \theta .
$$

Используя теперь (3), из (16) имеем

$$
\sigma(\theta)=\left(\frac{\left\langle\dot{\varepsilon}_{x}\right\rangle}{a}\right)^{\frac{1}{m}}|K|^{\frac{1}{m}-1} \cdot K
$$


где

$$
K=\cos ^{2} \theta+\frac{\left\langle\dot{\varepsilon}_{y}\right\rangle}{\left\langle\dot{\varepsilon}_{x}\right\rangle} \sin ^{2} \theta .
$$

Подставляя (17) в уравнения равновесия (8), (9), получаем систему уравнений

$$
\begin{gathered}
\left\langle\sigma_{x}\right\rangle=2 \int_{0}^{\frac{\pi}{2}}\left(\frac{\left\langle\dot{\varepsilon}_{x}\right\rangle}{a}\right)^{\frac{1}{m}}|K|^{\frac{1}{m}-1} \cdot K d \theta \\
\int_{0}^{\frac{\pi}{2}} K \cdot|K|^{\frac{1}{m}-1} \sin ^{3} \theta d \theta=0 .
\end{gathered}
$$

Уравнение (20) решается численно относительно величины $z=\left\langle\dot{\varepsilon}_{y}\right\rangle /\left\langle\dot{\varepsilon}_{x}\right\rangle$ $(-1 \leqslant z \leqslant 0)$, а затем из $(19)$ с учетом $(15)$ находится связь между микрои макропараметрами $a$ и $A$ :

$$
a=A\left[2 \int_{0}^{\frac{\pi}{2}}|K|^{\frac{1}{m}-1} \cdot K \cos ^{2} \theta \sin \theta d \theta\right]^{m} .
$$

4. Методика расчёта реологического деформирования нелинейно-упругого материала по структурной модели. Пусть к материальному объему («точке») в главных осях $O X Y Z$ приложены нормальные напряжения $\left\langle\sigma_{x}\right\rangle,\left\langle\sigma_{y}\right\rangle,\left\langle\sigma_{z}\right\rangle$.

Введём следующее обозначение:

$$
\begin{aligned}
K_{1}\left\{\left\langle\varepsilon_{x}\right\rangle,\left\langle\varepsilon_{y}\right\rangle,\left\langle\varepsilon_{z}\right\rangle, \theta, \varphi\right\}= \\
\quad=\left\langle\varepsilon_{x}(t)\right\rangle \cos ^{2} \theta+\left\langle\varepsilon_{y}(t)\right\rangle \sin ^{2} \theta \cos ^{2} \varphi+\left\langle\varepsilon_{z}(t)\right\rangle \sin ^{2} \theta \sin ^{2} \varphi
\end{aligned}
$$

$1^{\circ}$. Область упругости. Рассмотрим сначала методику расчёта в упругой области в момент приложения нагрузки $(t=0)$. Для этого, используя нелинейный закон упругости для структурного элемента (2), из (6) и (22) имеем $K_{1}=\left[\sigma(\theta, \varphi, 0)|\sigma(\theta, \varphi, 0)|^{n-1}\right] / E_{\mathrm{M}}$, откуда

$$
\sigma(\theta, \varphi, 0)=E_{\mathrm{M}}^{\frac{1}{n}} K_{1}\left|K_{1}\right|^{\frac{1}{n}-1} .
$$

Подставляя теперь (23) в (4), получаем при заданных $\left\langle\sigma_{x}\right\rangle,\left\langle\sigma_{y}\right\rangle,\left\langle\sigma_{z}\right\rangle$ (с учётом обозначения $(22))$ систему нелинейных уравнений относительно $\left\langle\varepsilon_{x}(0)\right\rangle$, $\left\langle\varepsilon_{y}(0)\right\rangle,\left\langle\varepsilon_{z}(0)\right\rangle$ :

$$
\begin{aligned}
& \left\langle\sigma_{x}\right\rangle=\frac{E_{\mathrm{M}}^{\frac{1}{n}}}{\pi} \int_{0}^{\pi / 2} \cos ^{2} \theta \sin \theta d \theta \int_{0}^{2 \pi} K_{1}\left|K_{1}\right|^{\frac{1}{n}-1} d \varphi, \\
& \left\langle\sigma_{y}\right\rangle=\frac{E_{\mathrm{M}}^{\frac{1}{n}}}{\pi} \int_{0}^{\pi / 2} \sin ^{3} \theta d \theta \int_{0}^{2 \pi} K_{1}\left|K_{1}\right|^{\frac{1}{n}-1} \cos ^{2} \varphi d \varphi, \\
& \left\langle\sigma_{z}\right\rangle=\frac{E_{\mathrm{M}}^{\frac{1}{n}}}{\pi} \int_{0}^{\pi / 2} \sin ^{3} \theta d \theta \int_{0}^{2 \pi} K_{1}\left|K_{1}\right|^{\frac{1}{n}-1} \sin ^{2} \varphi d \varphi .
\end{aligned}
$$

Система уравнений (24) решается численно.

64 
$2^{\circ}$. Область ползучести. Рассмотрим теперь ползучесть материала в главных осях при действии напряжений $\left\langle\sigma_{x}\right\rangle,\left\langle\sigma_{y}\right\rangle,\left\langle\sigma_{z}\right\rangle$ для $t>0$. Здесь из (1), (2) и (6) имеем

$$
\begin{aligned}
& \frac{1}{E_{\mathrm{M}}} \sigma(\theta, \varphi, t)|\sigma(\theta, \varphi, t)|^{n-1}+p(\theta, \varphi, t)= \\
& \quad=\left\langle\varepsilon_{x}(t)\right\rangle \cos ^{2} \theta+\left\langle\varepsilon_{y}(t)\right\rangle \sin ^{2} \theta \cos ^{2} \varphi+\left\langle\varepsilon_{z}(t)\right\rangle \sin ^{2} \theta \sin ^{2} \varphi
\end{aligned}
$$

Откуда

$$
\sigma(\theta, \varphi, t)=E_{\mathrm{M}}^{\frac{1}{n}} K_{2}\left|K_{2}\right|^{\frac{1}{n}-1}
$$

где

$$
\begin{aligned}
K_{2}\left\{\left\langle\varepsilon_{x}\right\rangle,\left\langle\varepsilon_{y}\right\rangle,\left\langle\varepsilon_{z}\right\rangle, \theta, \varphi, t\right\}=\left\langle\varepsilon_{x}(t)\right\rangle \cos ^{2} \theta & +\left\langle\varepsilon_{y}(t)\right\rangle \sin ^{2} \theta \cos ^{2} \varphi+ \\
& +\left\langle\varepsilon_{z}(t)\right\rangle \sin ^{2} \theta \sin ^{2} \varphi-p(\theta, \varphi, t)
\end{aligned}
$$

при этом в соответствии с (3) деформация ползучести в локальном элементе вычисляется в любой момент времени $t$ по формуле

$$
p(\theta, \varphi, t)=\int_{0}^{t} a \sigma(\theta, \varphi, t)|(\theta, \varphi, t)|^{m-1} d t
$$

Подставляя (26) в уравнение (4), с учетом обозначения (27) получаем систему трех нелинейны уравнений относительно $\left\langle\varepsilon_{x}\right\rangle,\left\langle\varepsilon_{y}\right\rangle,\left\langle\varepsilon_{z}\right\rangle$ при заданных $\left\langle\sigma_{x}\right\rangle,\left\langle\sigma_{y}\right\rangle,\left\langle\sigma_{z}\right\rangle$ :

$$
\begin{gathered}
\left\langle\sigma_{x}\right\rangle=\frac{E_{\mathrm{M}}^{\frac{1}{n}}}{\pi} \int_{0}^{\pi / 2} \cos ^{2} \theta \sin \theta d \theta \int_{0}^{2 \pi} K_{2}\left|K_{2}\right|^{\frac{1}{n}-1} d \varphi, \\
\left\langle\sigma_{y}\right\rangle=\frac{E_{\mathrm{M}}^{\frac{1}{n}}}{\pi} \int_{0}^{\pi / 2} \sin ^{3} \theta d \theta \int_{0}^{2 \pi} K_{2}\left|K_{2}\right|^{\frac{1}{n}-1} \cos ^{2} \varphi d \varphi, \\
\left\langle\sigma_{z}\right\rangle=\frac{E_{\mathrm{M}}^{\frac{1}{n}}}{\pi} \int_{0}^{\pi / 2} \sin ^{3} \theta d \theta \int_{0}^{2 \pi} K_{2}\left|K_{2}\right|^{\frac{1}{n}-1} \sin ^{2} \varphi d \varphi .
\end{gathered}
$$

Очевидно, что при $t=0$ величина $p(\theta, \varphi, 0)=0$ и система $(29)$ совпадает с (24).

Нелинейная система (29), так же как и система (24), решается относительно $\left\langle\varepsilon_{x}\right\rangle,\left\langle\varepsilon_{y}\right\rangle,\left\langle\varepsilon_{z}\right\rangle$ численно, тем самым определяется макронапряженнодеформированное состояние в процессе ползучести. Расчёт деформации ползучести осуществляется численно по хорошо известному методу: «шагами по времени».

Для исследования эффекта влияния деформации ползучести на компоненты вектора упругой деформации рассмотрим следующий режим нагружения: пусть при $t \in\left[0, t^{*}\right]$ действуют постоянные компоненты тензора напряжений $\left\langle\sigma_{x}\right\rangle,\left\langle\sigma_{y}\right\rangle,\left\langle\sigma_{z}\right\rangle$ (в главных осях), а в момент $t=t^{*}$ происходит полная разгрузка, т. е. $\left\langle\sigma_{x}\right\rangle=\left\langle\sigma_{y}\right\rangle=\left\langle\sigma_{z}\right\rangle=0$ для $t>t^{*}$. Тогда компоненты 
вектора упругой деформации при нагрузке $\ni^{\mathrm{H}}\left\{\left\langle e_{x}\right\rangle,\left\langle e_{y}\right\rangle,\left\langle e_{z}\right\rangle\right\}^{T}(T-$ операция транспонирования) в момент времени $t=0+0$ определяются из системы уравнений (24). Компоненты вектора упругих деформаций при разгрузке $\ni^{\mathrm{P}}\left\{\left\langle e_{x}\right\rangle,\left\langle e_{y}\right\rangle,\left\langle e_{z}\right\rangle\right\}^{T}$ в момент времени $t=t^{*}$ определяются из равенства

$$
\ni^{p}=\left(\begin{array}{c}
\left\langle\varepsilon_{x}\left(t^{*}-0\right)\right\rangle \\
\left\langle\varepsilon_{y}\left(t^{*}-0\right)\right\rangle \\
\left\langle\varepsilon_{z}\left(t^{*}-0\right)\right\rangle
\end{array}\right)-\left(\begin{array}{c}
\left\langle\varepsilon_{x}\left(t^{*}+0\right)\right\rangle \\
\left\langle\varepsilon_{y}\left(t^{*}+0\right)\right\rangle \\
\left\langle\varepsilon_{z}\left(t^{*}+0\right)\right\rangle
\end{array}\right),
$$

при этом величины $\left\langle\varepsilon_{i}\left(t^{*}-0\right)\right\rangle(i \equiv x, y, z)$ определяются из системы $(29)$ при $t=t^{*}$, в которой

$$
p\left(\theta, \varphi, t^{*}\right)=\int_{0}^{t^{*}} a \sigma(\theta, \varphi, t)|(\theta, \varphi, t)|^{m-1} d t,
$$

а величины $\left\langle\varepsilon_{i}\left(t^{*}+0\right)\right\rangle(i=x, y, z)$ также из системы $(29)$, но при $\left\langle\sigma_{x}\right\rangle=$ $=\left\langle\sigma_{y}\right\rangle=\left\langle\sigma_{z}\right\rangle=0$ при тех же значениях $p\left(\theta, \varphi, t^{*}\right)$. Тогда, если эффекта влияния ползучести на компоненты упругой деформации не наблюдается, должно выполняться условие

$$
\ni^{\mathrm{H}}=\ni^{p},
$$

если же этот эффект имеет место, то должно выполняться

$$
\ni^{\mathrm{H}} \neq \ni^{p} .
$$

5. Результаты расчётов и анализ результатов. Для оценки адекватности расчётов по структурной модели предварительно были выполнены расчёты в частном случае одноосного растяжения образца при $\left\langle\sigma_{x}\right\rangle \neq 0,\left\langle\sigma_{y}\right\rangle=\left\langle\sigma_{z}\right\rangle=0$ по программам нагружения, представленными в табл. 1. Макрохарактеристики материала приведены в табл. 2, и они соответствуют характеристикам компактной костной ткани в областях линейной $\left(0 \leqslant\left\langle\sigma_{x}\right\rangle \leqslant 53,56 \mathrm{MПа)} \mathrm{и}\right.$ нелинейной $\left(53,56 \leqslant\left\langle\sigma_{x}\right\rangle \leqslant 90,41 \mathrm{MПа)} \mathrm{ползучести}[1,2]\right.$. Значения параметров аппроксимации макрозависимостей (7) и (15) взяты из работы [7].

По вариантам $a_{1}$ и $a_{2}$ в экспериментах

Таблица 1 [1] проведено испытание костной ткани при

Программы нагружения

\begin{tabular}{c|c|c|c}
\hline № ва- & $\left\langle\sigma_{x}\right\rangle$, & $\left\langle\sigma_{y}\right\rangle$, & $\left\langle\sigma_{z}\right\rangle$, \\
рианта & МПа & МПа & МПа \\
\hline$a_{1}$ & 35,71 & 0 & 0 \\
$a_{2}$ & 53,56 & 0 & 0 \\
$a_{3}$ & 74,56 & 0 & 0 \\
$a_{4}$ & 90,41 & 0 & 0 \\
\hline
\end{tabular}
длительности нагружения 100 мин с последующей разгрузкой, а в вариантах $a_{3}$ и $a_{4}$ длительность испытаний составила 200 минут с последующей разгрузкой [2]. По приведенным программам и были выполнены расчёты по структурной модели. Результаты расчёта по разработанной методике значений упругой деформации костной ткани при нагрузке $\left(e^{\mathrm{H}}\right)$ и разгрузке после ползучести $\left(e^{\mathrm{p}}\right)$ приведены в табл. 3. Здесь же для сравнения приведены экспериментальные данные $[1,2]$, а также данные расчётов по обобщенной двухэлементной модели типа Максвелла, приведенные в работе [7].

Как следует из данных табл. 3, наблюдается хорошая коррелированность результатов расчёта по предложенной структурной модели с экспериментальными данными и данными расчёта по двухэлементной обобщенной модели Максвелла микронеоднородной среды [7]. 
Для исследования Таблица 2 эффекта влияния ползучести на мгновенноупругую деформацию при сложном напряженном состоянии был выполнен обстоятельный вариативный чисМеханические макрохарактеристики нелинейной упруго-

\begin{tabular}{c|c|c|c|c}
\multicolumn{4}{c|}{ сти и нелинейной вязкости костной ткани } \\
\hline $\begin{array}{c}\text { № ва- } \\
\text { рианта }\end{array}$ & $n$ & $\begin{array}{c}\langle E\rangle \\
\left(\mathrm{MПа}^{n}\right)\end{array}$ & $m$ & $\begin{array}{c}A \\
\left(\mathrm{MПа}^{-m}\right) \cdot \mathrm{мин}^{-1}\end{array}$ \\
\hline$a_{1}$ & 0,88 & 13020 & 1 & $1,516 \cdot 10^{-6}$ \\
$a_{2}$ & 0,88 & 13020 & 1 & $1,516 \cdot 10^{-6}$ \\
$a_{3}$ & 1,685 & 321060 & 8,455 & $2,05 \cdot 10^{-19}$ \\
$a_{4}$ & 1,685 & 321060 & 8,455 & $2,05 \cdot 10^{-19}$ \\
\hline
\end{tabular}
ленный анализ по структурной модели при различных значениях макропараметров $n, E, m$ и $A$ (см. $(7)$ и (15)). Для удобства графической иллюстрации полученных результатов рассмотрим подробно плоское напряженное состояние, когда $\left\langle\sigma_{x}\right\rangle \neq 0,\left\langle\sigma_{y}\right\rangle \neq$ $\neq 0,\left\langle\sigma_{z}\right\rangle=0$. Пусть напряжения в главных осях $\left\langle\sigma_{x}\right\rangle$ и $\left\langle\sigma_{y}\right\rangle$ удовлетворяют соотношению

$$
S=\sqrt{\left(\left\langle\sigma_{x}\right\rangle\right)^{2}+\left(\left\langle\sigma_{y}\right\rangle\right)^{2}-\left\langle\sigma_{x}\right\rangle\left\langle\sigma_{y}\right\rangle}=S_{0}=\mathrm{const},
$$

где $S$ - интенсивность напряжений.

В качестве примера приведем типичные результаты, полученные для пар значений $\left\{\left\langle\sigma_{x}\right\rangle,\left\langle\sigma_{y}\right\rangle\right\}$ при $S_{0}=55$ МПа для следующего режима нагружения: при $t \in[0,100]$ мин приложена пара напряжений $\left\{\left\langle\sigma_{x}\right\rangle,\left\langle\sigma_{y}\right\rangle\right\}$, удовлетворяющая $(34)$, а при $t=100$ мин производится разгрузка $\left(\left\langle\sigma_{x}\right\rangle=0,\left\langle\sigma_{y}\right\rangle=0\right)$ и в течение $t \in[100,400]$ мин развивается обратная ползучесть.

Результаты вариативных расчётов в зависимости от параметров $n, E, m$, $A$ показали, что годографы векторов $\ni^{\mathrm{H}}$ и $\ni^{\mathrm{P}}$ в пространстве главных деформаций не совпадают. В качестве примера для иллюстрации результатов расчёта на рис. 1 и 2 приведены проекции годографов векторов $\ni^{\text {н }}$ (сплошная линия) и $\ni^{\mathrm{p}}$ (штриховая линия) на плоскости $O\left\langle\varepsilon_{x}\right\rangle\left\langle\varepsilon_{y}\right\rangle$ для вариантов $b_{1}\left(n_{1}=0,6 ; E=13020(\mathrm{MП})^{n} ; m=2 ; A=3 \cdot 10^{-6}(\mathrm{MПа})^{-m}\right.$ мин $\left.^{-1}\right)$ и $c_{1}$ $\left(n_{1}=1,685 ; E=321060(\mathrm{MПа})^{n} ; m=8,455 ; A=2,5 \cdot 10^{-19}(\mathrm{MПа})^{\left.-m_{\mathrm{MuH}^{-1}}\right)}\right.$ соответственно.

Из этих рисунков видно, что при показателе нелинейности упругости $n<$ 1 проекция годографа вектора $\ni^{\text {н }}$ лежит внутри проекции годографа вектора $\ni^{\mathrm{p}}$, а при $n>1$ наблюдается обратная картина (при $n=1$, как показали расчёты, и сами годографы векторов $\ni^{\mathrm{H}}$ и $\ni^{\mathrm{p}}$, и их проекции совпадают). На рис. 1,2 отрезками соединены точки, соответствующие одним и тем же парам

Таблица 3

Экспериментальные и расчётные значения упругой деформации костной ткани при нагрузке $e^{\mathrm{H}}$ и разгрузке $e^{\mathrm{p}}$ после ползучести

\begin{tabular}{|c|c|c|c|c|c|c|c|}
\hline \multirow[b]{2}{*}{$\begin{array}{c}\text { № } \\
\text { вариан- } \\
\text { та }\end{array}$} & \multirow[b]{2}{*}{$\begin{array}{l}\left\langle\sigma_{x}\right\rangle \\
\text { МПа }\end{array}$} & \multicolumn{3}{|c|}{$e^{\mathrm{H}}, \%$} & \multicolumn{3}{|c|}{$e^{\mathrm{P}}, \%$} \\
\hline & & $\begin{array}{l}\text { Экспе- } \\
\text { римент }\end{array}$ & $\begin{array}{c}\text { Расчёт } \\
\text { по } \\
\text { структур- } \\
\text { ной } \\
\text { модели }\end{array}$ & $\begin{array}{c}\text { Расчёт } \\
\text { по } \\
\text { обобщен- } \\
\text { ной } \\
\text { модели } \\
\text { Максвелла }\end{array}$ & $\begin{array}{l}\text { Экспе- } \\
\text { римент }\end{array}$ & $\begin{array}{c}\text { Расчёт } \\
\text { по } \\
\text { структур- } \\
\text { ной } \\
\text { модели }\end{array}$ & $\begin{array}{c}\text { Расчёт } \\
\text { по } \\
\text { обобщен- } \\
\text { ной } \\
\text { модели } \\
\text { Максвелла }\end{array}$ \\
\hline$a_{1}$ & 35,71 & 0,178 & 0,178 & 0,178 & 0,152 & 0,159 & 0,162 \\
\hline$a_{2}$ & 53,56 & 0,255 & 0,255 & 0,250 & 0,245 & 0,240 & 0,236 \\
\hline$a_{3}$ & 74,56 & 0,470 & 0,445 & 0,455 & 0,670 & 0,638 & 0,642 \\
\hline$a_{4}$ & 90,41 & 0,570 & 0,616 & 0,570 & - & - & - \\
\hline
\end{tabular}




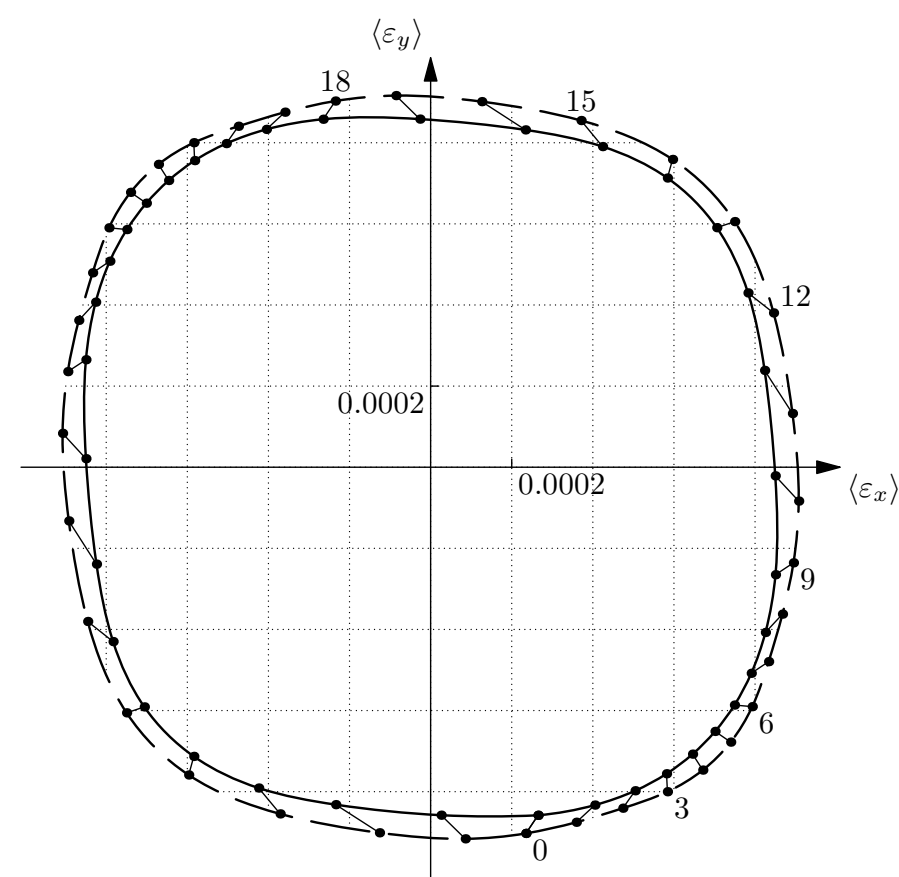

Рис. 1. Значения компонент проекции вектора упругой деформации $\ni^{\text {н }}$ (сплошная линия) на плоскость $0\left\langle\varepsilon_{x}\right\rangle\left\langle\varepsilon_{y}\right\rangle$ в момент приложения нагрузки $(t=0)$ и компонент проекции вектора $\ni^{\mathrm{P}}$ (штриховая линия) после ползучести в течение 100 мин при значениях $\left\langle\sigma_{x}\right\rangle$ и $\left\langle\sigma_{y}\right\rangle$, удовлетворяющих условию (34); вариант $b_{1}$

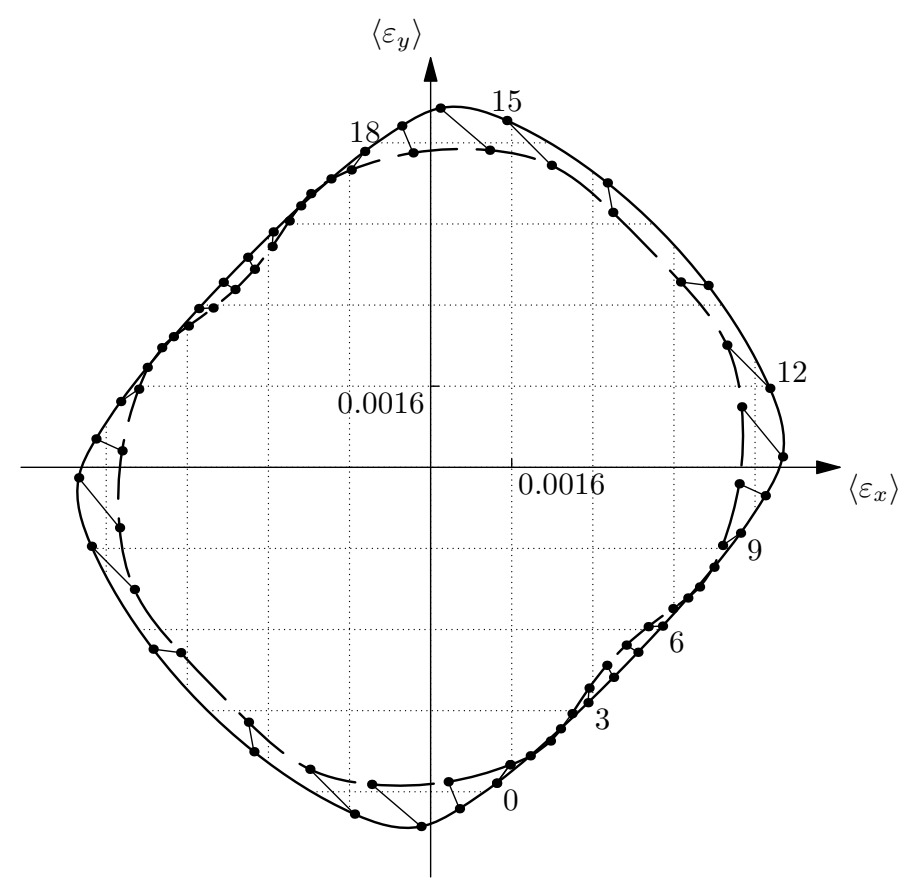

Рис. 2. Значения компонент проекции вектора упругой деформации $\ni^{\text {н }}$ (сплошная линия) на плоскость $0\left\langle\varepsilon_{x}\right\rangle\left\langle\varepsilon_{y}\right\rangle$ в момент приложения нагрузки $(t=0)$ и компонент проекции вектора $\ni^{\mathrm{P}}$ (штриховая линия) после ползучести в течение 100 мин при значениях $\left\langle\sigma_{x}\right\rangle$ и $\left\langle\sigma_{y}\right\rangle$, удовлетворяющих условию (34); вариант $c_{1}$ 
значений $\left\langle\sigma_{x}\right\rangle,\left\langle\sigma_{y}\right\rangle$, откуда наглядно (визуально) видно, что действительно вектор упругих деформаций при $n \neq 1$ в процессе ползучести изменяет не только свой модуль, но и направление в пространстве главных деформаций.

Другой важный факт, установленный расчётами, состоит в том, что в процессе ползучести после разгрузки (вследствие релаксации остаточных микронапряжений в локальных элементах структурной модели) происходит восстановление первоначальных упругих свойств, т. е. годограф вектора $\ni^{\mathrm{p}}$ приближается к годографу $\ni^{\text {н }}$, и в предельном случае (при $t \rightarrow \infty$ ) эти годографы совпадают. Другими словами, мгновенная нелинейно-упругая деформация проявляет одновременно наследственные свойства и свойства механической памяти.

Этот факт имеет большое значение при построение феноменологических макромоделей ползучести нелинейно-упругих материалов, поскольку для известных «классических» (линейно-упругих) материалов используется гипотеза отделимости упругой деформации и реологической деформации, что с математической точки приводит к аддитивности упругой деформации и деформации ползучести. Для рассматриваемого класса нелинейно-упругих материалов эту гипотезу применять нельзя, поскольку упругая деформация неявно зависит от времени (при постоянном тензоре напряжений!) через накопленную деформацию ползучести. Поэтому в данном случае уравнения состояния будут иметь более сложный характер.

\section{Выводы.}

1. Показано, что эффект влияния ползучести на упругую деформацию имеет место и при сложном напряженном состоянии в случае, если локальные элементы (и материал на макроуровне) наделены законом нелинейной упругости. При этом при степенной аппроксимации закона нелинейной упругости наблюдается принципиально различный характер годографов векторов упругой нагрузки и разгрузки после ползучести в пространстве главных деформаций при показателях нелинейности $n>1$ и $n<1$ (при $n=1$ этого эффекта не наблюдается).

2. Показано, что происходит не только изменение модуля вектора нелинейных упругих деформаций в пространстве деформаций вследствие ползучести, но и его поворот, что является новым, неизвестным ранее фактом.

3. Установлено, что вследствие ползучести компоненты нелинейно-упругой деформации проявляют одновременно свойства механической памяти, поскольку в процессе ползучести после разгрузки происходит полное эволюционное восстановление первоначальных упругих свойств, а также вязкоупругости, так как мгновенно-упругая деформация неявно зависит от времени. Это означает, что на феноменологическом уровне механики сплошных сред нельзя использовать гипотезу аддитивности упругой деформации и деформации ползучести.

Работа выполнена при поддержке РФФИ (код проекта 10-01-00644-а).

\section{БИБЛИОГРАФИЧЕСКИЙ СПИСОК}

1. Кнетс И. В., Вилкс Ю.К. Ползучесть компактной костной ткани человека при растяжении // Механика полимеров, 1975. - Т. 11, № 4. - С. 634-638; англ. пер.: Knets I. V., Vilks Yu. K. Creep of compact human bony tissue under tension // Mechanics of Composite Materials, 1975. - Vol. 11, No. 4. - P. 543-547. 
2. Мелнис А. Э., Лайзан Я. Б. Нелинейная ползучесть компактной костной ткани человека при растяжении // Механика полимеров, 1978. - Т.14, №1. - С. 97-100; англ. пер.: Melnis A. E., Laizan Ya. B. Nonlinear creep of human compact bone tissue upon stretching // Mechanics of Composite Materials, 1978. - Vol. 14, No. 1. — P. 82-84.

3. Самарин Ю. П. О применении теории управления к исследованию ползучести и конструкций / В сб.: Механика деформируемых сред. - Куйбышев: КГУ, 1976. - С. 123-129.

4. Радченко В. П., Самарин Ю. П. Влияние ползучести на величину упругой деформации слоистого композита // Механика композитных материалов, 1983. - №2 - - С. 231237; англ. пер.: Radchenko V.P., Samarin Yu.P. Effect of creep on the elastic deformation of a laminar composite// Mechanics of Composite Materials, 1983. - Vol.19, No. 2. P. $162-168$.

5. Радченко В. П. Об одной структурной реологической модели нелинейно-упругого материала // Прикладная механика, 1990. - Т. 26, № 6. - С. 67-74; англ. пер.: Radchenko V.P. Structural rheological model of a nonlinearly elastic material // Sov. Appl. Mech., 1990. Vol. 26, No. 6. - P. 577-582.

6. Радченко В. П., Шапиевский Д. В. О дрейфе упругой деформации для нелинейно-упругих материалов вследствие ползучести // Вестн. Сам. гос. техн. ун-та. Сер.: Физ.мат. науки, 2006. - № 43. - С. 99-106.

7. Радченко В.П., Шапиевский Д. В. Математическая модель ползучести микронеоднородного нелинейно-упругого материала // ПМТФ, 2008. - Т. 49, № 33. - С. 157-163; англ. пер.: Radchenko V. P., Shapievskii D. V. Mathematical model of creep for a microinhomogeneous nonlinearly elastic material // J. Appl. Mech. Tech. Phys., 2008. - Vol.49, No. 3. - P. 478-483.

8. Радченко В. П., Андреева E. А. О дрейфе и эффекте памяти нелинейно-упругой деформации вследствие ползучести для микронеоднородных материалов в условиях одноосного напряженного состояния // Вестн. Сам. гос. техн. ун-та. Сер. Физ.-мат. науки, 2009. - № 2(19). - С. 72-77.

9. Радченко В. П., Ерёмин Ю. А. Реологическое деформирование и разрушение материалов и элементов конструкций. - М.: Машиностроение-1, 2004. - 264 с.

Поступила в редакцию 08/IX/2009;

в окончательном варианте - 13/II/2010.

MSC: 74E35, 74B20, 74A20

\section{CREEP STRUCTURAL MODEL OF NONLINEARLY ELASTIC MICROHETEROGENEOUS MATERIAL IN COMPLEX STRESS \\ STATE}

\section{P. Radchenko, E. A. Andreeva, A. V. Nikishaev}

Samara State Technical University,

244, Molodogvardeyskaya str., Samara, 443100.

E-mails: radch@samgtu.ru, andreevaSamara25@yandex.ru, anton.nik@gmail.com

Structural model for microheterogeneous nonlinear elastic material for the description of creep under complex stress condition. It is shown that for this class of materials the effect of creep deformation on the elastic deformation is observed. This is not only a change in the modulus of nonlinearly elastic deformations in the space of deformations due to creep, but its turn. The results of variant calculations are presented, their analysis is carried out.

Key words: nonlinearly elastic material, creep, structural model, complex stress state, the drift of the elastic deformation.

Original article submitted 08/IX/2009; revision submitted $13 / \mathrm{II} / 2010$.

Vladimir P. Radchenko (Dr. Sci. (Phys. \& Math.)), Head of Dept., Dept. of Applied Mathematics \& Computer Science. Elena A. Andreeva, Postgraduate Student, Dept. of Applied Mathematics \& Computer Science. Anton V. Nikishaev, Software Engineer, Dept. of Applied Mathematics \& Computer Science. 